\title{
Desempeño laboral en instituciones de educación superior: una revisión
} Latinoamericana de literatura

\section{Job performance in higher education institutions: A Latin American literature review}

\author{
${ }^{1}$ Universidad Cesar Vallejo, Perú. \\ ${ }^{a}$ pamasifuenb@ucvvirtual.edu.pe \\ b jgarater@ucvvirtual.edu.pe \\ c dpalominoal@ucvvirtual.edu.pe
}

Beto Pashanasi Amasifuen ${ }^{1, a}$ 0000-0002-5023-1849

Jhonny Gárate Ríos ${ }^{1, b}$ 0000-0002-3062-6106

Gabriela del Pilar Palomino Alvarado ${ }^{1, \mathrm{c}} \underline{\text { 0000-0002-2126-2769 }}$
Recibido: $16 / 05 / 2021$
Aprobado: $25 / 08 / 2021$
Publicado: $15 / 09 / 2021$

\begin{abstract}
Work performance is being studied in areas ranging from work satisfaction to organizational climate. This situation has distorted it into a subject that is not approached as main goal, but associated to different factors, such as: effectiveness, adaptability to work, communication, interpersonal relationships, administrative management, work efficiency. Due to this, the aim is to analyze Latin American scientific articles, which are published in ScieLO, EBSCO, ScienceDirect, ProQuest, Latindex, REDIB, Redalyc, CLASE, DOAJ, ROAD and Dialnet databases, from the years 2015 to 2021, based on topics associated with work performance. In this research, a systematic bibliographic review of 27 articles from Latin American countries was carried out. As highlighted results, the most studied topics are those related to teamwork linked to efficiency, achievement orientation and those related to the aforementioned factors that influence and improve work performance.
\end{abstract}

Keywords: training; management; efficiency; effectiveness; university.

Palabras clave: capacitación; gestión; eficiencia; efectividad; universidad. 


\section{Introducción}

En la actualidad, las evaluaciones del desempeño laboral (DL) en instituciones públicas, deben responder a identificación de brechas de competencias en personal administrativo según perfil de puesto, para diseñar estrategias de fortalecimiento y mejora, que promuevan logros de metas y/o resultados planificados (Álvarez-Indacochea et al., 2018). La gestión de instituciones de educación superior (IES) difiere de organizaciones empresariales, debido al índole del entorno universitario, que por tareas, roles y responsabilidades de trabajadores difieren a otras organizaciones (Pongton \& Suntrayuth, 2019).

Considerando, enlaactualidadesmás difícilconseguir que empleados transformen comportamientos enmarcadas en antiguos paradigmas y venzan los retos de adaptación, se hace indispensable el manejo de técnicas y procedimientos que cambian de momento, concibiendo de forma obligatoria adecuar procesos en el desarrollo de instituciones (Cubero et al., 2018). Mientras que, no basta con evaluar los recursos humanos desde el punto de vista cognitivo e intelectual, sino debemos ir más allá identificando y fortaleciendo habilidades, y potencialidades propias de cada servidor (Fulthorp \& D'Eloia, 2015).

Por esa razón, una de las principales inquietudes de los responsables del manejo del recurso humano se encuentra en disponer de empleados con alto nivel de satisfacción en su trabajo, con motivación, más productivos y comprometidos (Pa'wan \& Omar, 2018; Rodríguez et al., 2015). Es por ello, que cuando un trabajador muestra satisfacción en las tareas que desarrolla es un pronóstico significativo del DL (Eirín et al., 2020).

Por otro lado, el clima organizacional se encarga de estudiar los comportamientos, actitudes y desempeño humano en una empresa; basándose en teorías, métodos y principios de la psicología, la sociología y la antropología cultural (Ivancevich et al., 2006). Otros mencionan que el clima organizacional es la atención de la aplicación de las fortalezas y las capacidades psicológicas de los gestores del talento humano, los cuales se cuantifican, se fomentan y se administran de manera eficaz (Chiavenato, 2009). Es así que, el logro o el fracaso que se presenta en las IES es debido al clima organizacional, liderazgo y relaciones interpersonales (Musah et al., 2016).
Es por ello, que el DL como construcción de una teoría, está comprendida por conductas que se encuentran dentro de la observación de los empleados que coadyuvan al logro de los resultados (Campbell \& Wiernik, 2015). Por consiguiente, el análisis y la evaluación del DL es una fortaleza en distintas actividades de las instituciones, que permiten optimizar la elección del personal, otorgar compensaciones y retribuciones o fortalecimiento de capacidades (Ramos-Villagrasa et al., 2019). Sin embargo, es importante considerar el equilibrio entre el trabajo y la familia que comúnmente se encuentran en conflicto, para ello se pueden implementar políticas que se orienten a dar una solución a los conflictos entre trabajo y familia (Medina-Garrido et al., 2017).

Asimismo, es importante conocer el DL, desde la salud, capacitación, satisfacción laboral y clima organizacional de los trabajadores en una institución de educación superior. Sobre el particular, Khan et al. (2019) mencionan que mediante el fortalecimiento de capacidades se puede mejorar el DL de los empleados, por consiguiente, coadyuva en el cumplimiento de los objetivos. Por otro lado, Al-Kurdi et al. (2020) menciona que no debe dejarse de lado lo potente que puede ser la gestión del conocimiento.

En esa misma línea, referente a la salud, Foy et al. (2019) afirma que el estrés laboral es un factor que genera disminución en el desempeño laboral. Por otro lado, las IES están cambiando, por la aparición del modelo basado en la perfección de los resultados, por lo que Grobler \& Van Rensburg (2019) indican que dichos cambios han determinado la forma en que los empleados ven su ambiente laboral.

Del mismo modo, para que los empleados estén motivados, debe haber un apropiado ambiente laboral que origine tranquilidad y se enfoque en la confianza (Bohórquez et al., 2020). En este sentido, el ambiente laboral debe ofrecer seguridad a los trabajadores, por el contrario, la inseguridad puede producir una disminución del rendimiento laboral (Sora et al., 2021).

De igual manera, las consecuencias de la globalización obligan a los tomadores de decisión asegurar la sostenibilidad futura de las instituciones (Mohd Ali et al., 2019), debido a ello las IES requieren una transformación si desean sobrevivir y crecer en este escenario competitivo (Dermawan \& Handayani, 2019). 
Es preciso señalar, que el DL de los trabajadores es importante para su valoración propia y para su logro, para cual el individuo debe estar satisfecho en su centro laboral. En virtud de ello, Chiang y Ojeda (2013), define a la satisfacción laboral como el sentir negativo o positivo que las personas tienen en relación a las actividades que desarrollan en su centro laboral. De la misma manera, Zapata y Rueda (2018) concibe la satisfacción laboral como la posición o la actitud en que un trabajador o empleado se hace cargo del trabajo. En relación a ello, Jyoti \& Sharma (2017) afirma que su necesidad y sus aspiraciones son distintos y dificultoso de comprender.

En esa misma línea, la satisfacción laboral es un proceso que se crea implementando estrategias de mejoras salariales, disponibilidad de equipos tecnológicos para desarrollar sus funciones, otorgamiento de ascensos al buen desempeño y buena praxis organizacional (Vargas y Flores, 2019). Asimismo, Pujol-Cols y Dabos (2018) lo define como una conjugación de pensamientos y sentimientos.

Finalmente, el objetivo que se pretende alcanzar es analizar los temas fundamentales que se estudian respecto al DL en Latinoamérica. La nueva realidad nacional e internacional y la demanda creciente para el desarrollo personal de los jóvenes obligan a las IES, como los institutos y las universidades, evaluar e innovar la gestión administrativa para mejorar su desempeño.

\section{Metodología}

Continuando con el estudio, se presenta en forma detallada los procesos que se realizaron para el desarrollo de la presente investigación.
El estudio se basó en el análisis sistemático de la literatura (Reyes, 2020) y para obtener los artículos científicos se realizó la búsqueda en algunas bases de datos disponibles durante el tiempo de 30 días. Asimismo, se realizó el análisis de los datos tomando en consideración los componentes que se utilizan en las revisiones sistemáticas y metanálisis propuestas por (Moher et al., 2014), para ello se incluyó investigaciones que involucra a literatura teórica, seleccionando estudios originales de revistas científicas, para el cual se tuvieron en cuenta los criterios siguientes: 1) bases de datos ScieLO, EBSCO, Science Direct, ProQuest, Latindex, REDIB, Redalyc, CLASE, DOAJ, ROAD y Dialnet; 2) utilizando descriptores "desempeño laboral" AND "universidad", "clima organizacional" AND "universidad", "satisfacción laboral" AND “universidad”; 3) estudios científicos arbitrados y académicos de los últimos 5 años y 4) en idioma español o castellano (Sánchez, 2011); realizando en una primera etapa la lectura de los títulos y resúmenes en los estudios encontrados, seleccionando los potenciales elegidos; y en la segunda etapa se hizo la lectura de todo el texto de los seleccionados para corroborar su elegibilidad.

A continuación, se descartaron 10 artículos duplicados, ya que algunas revistas publican los artículos en dos o más bases de datos; quedando un total de 46 registros. Continuando con el proceso de elegibilidad se excluyeron 12 referencias que no cumplían con los criterios definidos para el título y resumen. Los 34 artículos científicos que resultaron fueron leídos para confirmar la elección. Finalmente, se descartaron 7 artículos de acuerdo a motivos, quedando 27 artículos para la realización del análisis sistemático, como se observa en la Figura 1. 


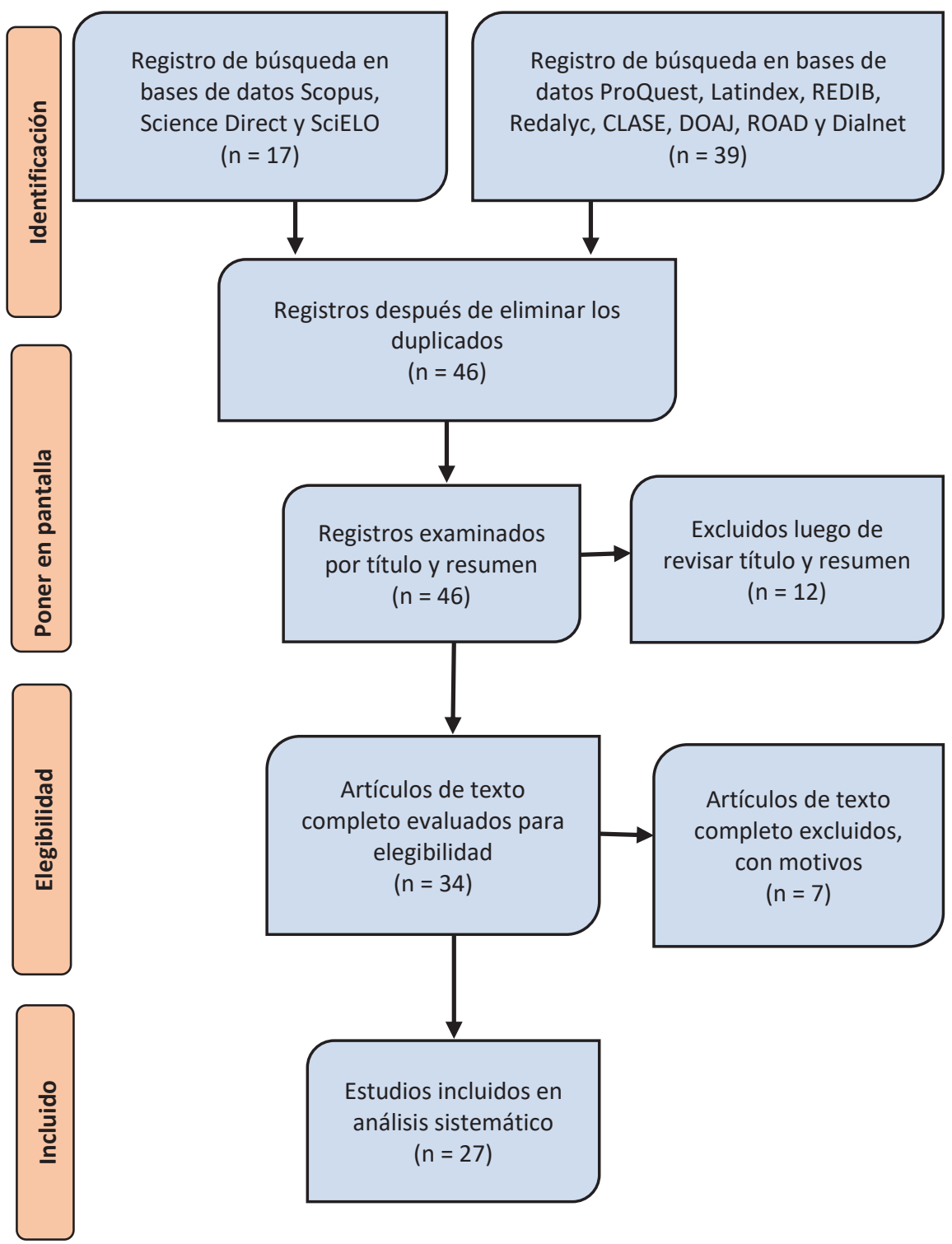

Figura 1. Resultados de búsqueda y filtros aplicados Fuente: adaptado de Moher et al. (2014).

De acuerdo a los criterios descritos en esta sección, se escogieron 6 artículos de Perú, 3 de Ecuador, 3 de Colombia, 3 de México y 1 de Chile; haciendo un total de 16 artículos. Para una mejor revisión se organizaron de acuerdo a su procedencia y se procesó a través de una matriz de construcción propia, donde se trabajaron los rangos de autores del estudio y año de publicación, título o tema de la investigación y los principales resultados y aportes que se encontraron relacionados con el DL. Una vez culminada la matriz con los 15 artículos se identificó los temas investigados, así como los aportes o propuestas que se presentan; siendo estos categorizados en un total de 12 , los mismos que se estructuraron de acuerdo a la regularidad con la que repetían en los estudios.
Por consiguiente, para elegir los aportes o propuestas se examinaron todas las ideas y afirmaciones que coadyuvaron al conocimiento teórico o al mejoramiento del DL. Los resultados se ordenaron a través de un histograma de barras con los temas categorizados y a través de una tabla donde se sintetiza la repetición de las aportes o propuestas que se llevan a cabo en los artículos revisados.

\section{Resultados}

De acuerdo a lo descrito en la sección metodología, los resultados se presentaron a través de un diagrama de barras con los temas categorizados y a través de una tabla donde se sintetiza la repetición 
de las aportes o propuestas que se llevan a cabo en los artículos revisados.

\section{Temas fundamentales de estudio sobre el desempeño laboral}

De acuerdo a los artículos que se seleccionaron para el presente estudio, se han conseguido diferentes temas de investigación, los mismos que se pueden hallar en los estudios revisados (ver Figura 2).

La hermenéutica de los resultados se estará realizando desde las categorías que tiene una cantidad menor de artículos y seguiremos a los aquellas que tienen más, es decir iremos de menor a mayor. Es importante indicar que este proceso utiliza el concepto de cantidad y no de relevancia, ya que este apartado no intenta realizar una valoración de la envergadura de los temas; sino mostrarlos de manera ordenada que nos permita la máxima comprensión.

Como podemos observar en la Figura 2 existe un tema "las cualidades perfeccionadas impactan en el DL" estudiado solamente por un artículo. En esta investigación se indica que el perfeccionamiento continuo de las cualidades de los servidores de una institución de educación superior logra un impacto en el DL (Álvarez-Indacochea et al., 2018). Asimismo, se puede corroborar en la Figura 1 el tema "la perfección continua de las destrezas", la misma que se orienta a la optimización continua de las habilidades, mejorando el DL de los trabajadores en las IES (Álvarez-Indacochea et al., 2018).

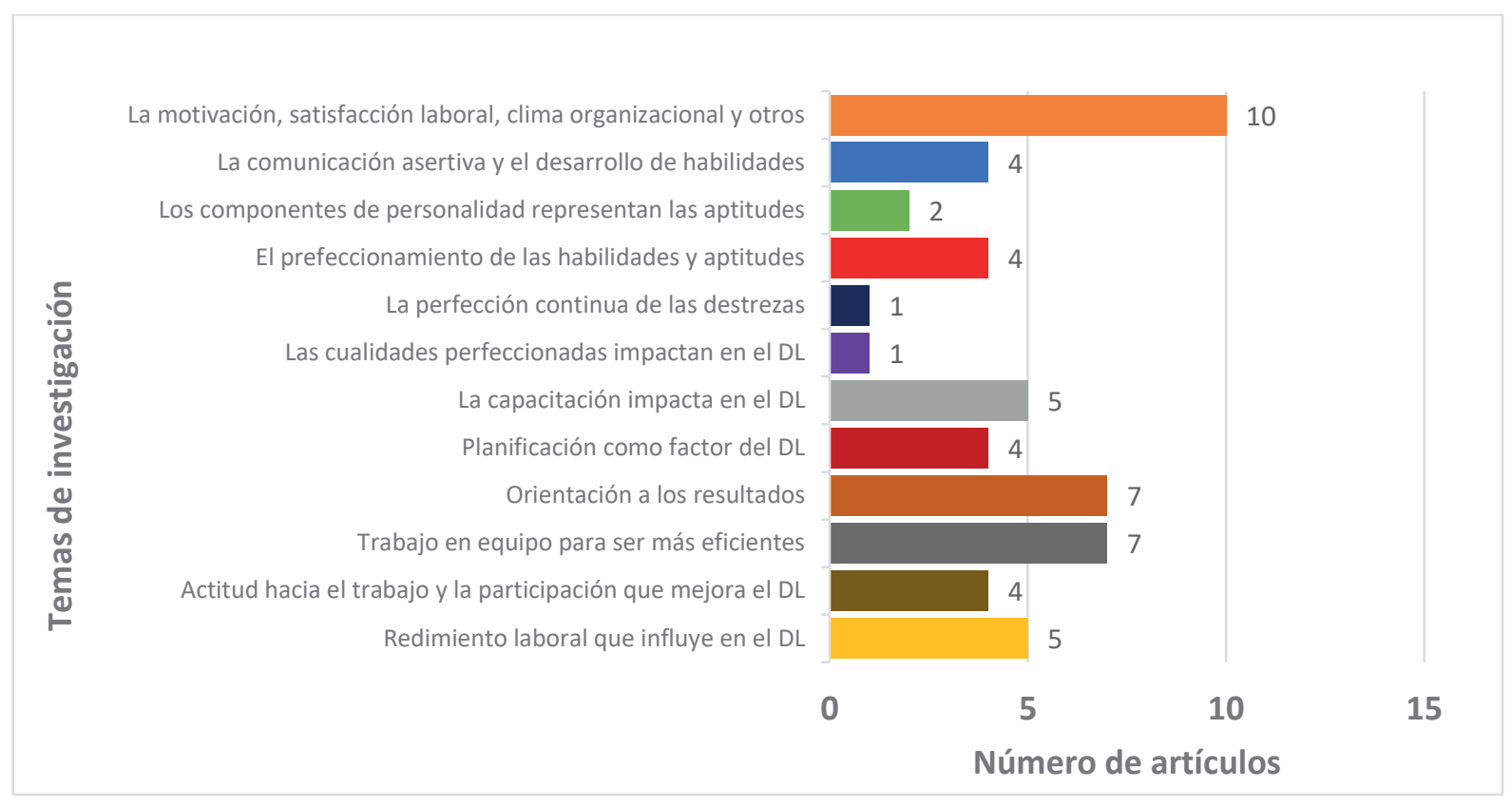

Figura 2. Categorías conseguidas de los temas de investigación sobre desempeño laboral.

Continuando, en la Figura 2 podemos constatar un tema "los componentes de personalidad representan las aptitudes" que fueron estudiados en dos artículos. Los estudios tienen como finalidad determinar la influencia de la inteligencia emocional sobre las competencias laborales y como se relaciona el clima organizacional y la violencia laboral. A saber: a) la aptitud personal representada por el reconocimiento de emociones influye significativamente en las competencias laborales (Duque et al., 2017); y b) las aptitudes representadas por el nivel educativo no hay diferencias significativas favorables dentro del clima organizacional (Pantoja-Pantoja et al., 2020).
Asimismo, en la Figura 2 se puede evidenciar que cuatro temas han sido investigados en 4 artículos cada uno. Los temas fueron: "actitud hacia el trabajo y la participación que mejoran el DL", "planificación como factor del DL", "el perfeccionamiento de las habilidades y aptitudes" y "la comunicación asertiva y el desarrollo de habilidades".

De este modo, las investigaciones categorizadas dentro de "actitud hacia el trabajo y la participación que mejoran el DL", se orientaban al clima organizacional y el rendimiento laboral, la motivación y el DL, y cultura organizacional y 
satisfacción laboral. A conocer: a) el cargo laboral, la modalidad de contratación y la edad influyen en la actitud de los trabajadores hacia el trabajo (Calizaya y Bellido, 2019); b) las opiniones, las ideas y los pensamientos deben de tomarse en cuenta para promover la participación de los trabajadores (Orbe-Guaraca y Ordoñez-Espinoza, 2018); c) la responsabilidad e identificación con la labor encomendada deben ser valorados (Rodríguez-Marulanda y Lechuga-Cardozo, 2019) y d) la actitud amable y la calidad para brindar el servicio al usuario permite realizar de forma adecuada las actividades y funciones asignadas (Vargas y Flores, 2019).

Por otro lado, la siguiente categoría "planificación como factor del DL", contaba con cuatro artículos que explican la planificación como motor de éxito del desempeño laboral. En un artículo refieren que la disposición a la planificación por parte de los trabajadores se relaciona con la modalidad de vínculo laboral, más no con la edad y muy bajo con el cargo laboral (Calizaya y Bellido, 2019); otro refiere que no se debe improvisar actividades de trabajo y no realizarlo sin antes haber planificado (Rodríguez-Marulanda y Lechuga-Cardozo, 2019); en el tercero hace referencia a la planificación de gestión administrativa que coadyuva a mejorar el DL (Panduro et al., 2019). Finalmente, el cuarto artículo describe la importancia de conocer la misión (Vargas y Flores, 2019).

Por otra parte, la categoría "el perfeccionamiento de las habilidades y aptitudes", los artículos tienen la finalidad de dar a conocer la representación del reconocimiento de emociones en la aptitud personal y limitaciones para mejorar habilidades; en consecuencia los estudios que representan a esta categoría desarrollan los siguientes puntos: 1) el perfeccionamiento continuo de las habilidades y aptitudes de los trabajadores de las instituciones (Álvarezetal., 2018);2) la aptitud social representada por la comunicación asertiva y desarrollo de habilidades influyen en las competencias laborales (Duque et al., 2017); 3) el capital humano valorado favorablemente en las IES, especialmente en los aspectos de habilidades y conocimientos (Pedraza, 2020); y 4) el reconocimiento de habilidades y capacidades de trabajadores brinda oportunidades de perfeccionarlos (Bravo et al., 2021).

En cuanto al tema "la comunicación asertiva y el desarrollo de habilidades" destaca su importancia e incidencia en el rendimiento laboral de trabajadores. En relación a esta categoría, un artículo determina la comunicación y su incidencia en el DL del trabajador (Hernández y Morales, 2017); otro artículo establece la aptitud social que se representa por la comunicación asertiva y desarrollo de habilidades e influencia en las competencias laborales (Duque et al., 2017); un tercer artículo señala la existencia de mínimo conocimiento para el desarrollo de tareas encomendadas (Rodríguez-Marulanda y LechugaCardozo, 2019); y el cuarto artículo precisa que el desarrollo de habilidades digitales y tecnológicas contribuye a la ética profesional (Ticona, 2020).

Del mismo modo, en la Figura 2 se logra examinar que cinco temas son investigados en 2 artículos cada uno, estos son: "rendimiento laboral que influye en el DL" y "la capacitación impacta en el DL".

En relación a la categoría "rendimiento laboral que influye en el DL", presenta diferentes aspectos de relación entre rendimiento de los trabajadores y entorno laboral. Por ello que, los estudios se encargan de investigar los siguientes aspectos: 1) el cargo laboral, el género y la edad y su influencia con el rendimiento laboral y por consiguiente en el DL (Calizaya y Bellido, 2019); 2) ambiente laboral para realizar tareas, como elemento influyente del rendimiento de los trabajadores (Orbe-Guaraca y Ordoñez-Espinoza, 2018); 3) calidad de procesos y servicios, formación de alumnos, vinculación con sector productivo y sociedad en general; visto como resultados del rendimiento y desempeño laboral en IES (Pedraza, 2020); 4) gestión administrativa y relación con el rendimiento laboral e influencia del DL (Panduro et al., 2019); y 5) buena colaboración entre trabajadores mejora la eficacia y rendimiento futuro (Ticona, 2020).

Con respecto a la categoría "la capacitación impacta en el DL", los estudios destacan la importancia del fortalecimiento de capacidades en trabajadores. Referente a esto: un artículo menciona que perfeccionar continuamente las capacidades fortalece el desarrollo de la institución (Álvarez et al., 2018); otro artículo da a conocer que, capacitación en gestión universitaria mejora el DL (Chávez et al., 2018); el tercer artículo alude a la importancia del respaldo de jefes inmediatos cuando trabajadores tengan que asistir a capacitación (Orbe-Guaraca y Ordoñez-Espinoza, 2018); el cuarto artículo afirma que el impacto de capacitaciones en algunos trabajadores es alto, en otros es término medio y varios no muestran 
diferencias (Rodríguez-Marulanda y LechugaCardozo, 2019); y el quinto artículo asegura que capacitaciones es factor de motivación que influye en la satisfacción laboral y por ende en el DL (Rojas y Távara, 2017).

Asimismo, en la Figura 2 se corrobora dos temas que se han estudiado en siete artículos cada uno, siendo el primero "Trabajo en equipo para ser más eficientes" y el segundo "Orientación a los resultados".

Referente a la categoría "trabajo en equipo para ser más eficientes", las investigaciones tienen objeto de evaluar la importancia del trabajo conjunto y colaborativo en IES. Dentro de ello se destaca: 1) disponibilidad al trabajo en equipo relacionada con el cargo laboral, modalidad de contratación y edad (Calizaya y Bellido, 2019); 2) aptitud personal representada por trabajo en equipo influye en competencias laborales (Duque et al., 2017), logrando la motivación y satisfacción laboral (Hernández y Morales, 2017; Rojas y Távara, 2017); 3) logro de la meta y conocimiento se complementan con relaciones interpersonales para ejecutar las labores (Panduro et al., 2019; Rodríguez-Marulanda y Lechuga-Cardozo, 2019); y 4) convivencias digitales y la tecnología son oportunidades de coordinación y vinculación que ofrecen las redes digitales (Ticona, 2020).

En cuanto a la categoría "orientación a los resultados", los estudios describen la disposición de trabajadores para lograr metas propuestas. Estos son: 1) predisposición al cumplimiento de objetivos relacionada al cargo laboral, tipo de contrato y edad (Calizaya y Bellido, 2019); 2) aptitud personal representada por orientación al logro, influye en las competencias laborales (Duque et al., 2017); 3) estado de ánimo influye en el cumplimiento de actividades diarias (Orbe-Guaraca y OrdoñezEspinoza, 2018); 4) orientación al logro de la meta no sacrifica la calidad del trabajo (RodríguezMarulanda y Lechuga-Cardozo, 2019); 5) la ejecución de gestión administrativa es pasar a la acción con el fin de orientar a los objetivos (Panduro et al., 2019); 6) orientación a los resultados de trabajadores se logra con comunicación efectiva y colaboración (Ticona, 2020); y 7) satisfacción laboral de trabajadores es logro de resultados (Vargas y Flores, 2019). organizacional y otros factores", donde se juntó a factores que no se mencionan anteriormente. Dentro de ellos se puede mencionar los siguientes: a) la motivación (Bravo et al., 2021; Hernández y Morales, 2017; Orbe-Guaraca y Ordoñez-Espinoza, 2018; Rojas y Távara, 2017; Zuta et al., 2018); b) clima organizacional (Montoya et al., 2017; Pantoja-Pantoja et al., 2020); c) satisfacción laboral (Bravo et al., 2021; Pedraza, 2020; Rojas y Távara, 2017; Ticona, 2020) y d) cultura organizacional (Vargas y Flores, 2019).

En resumen, se logra señalar que los temas que más se investigaron en referencia al DL son: 1) la motivación, satisfacción laboral, clima organizacional y otros; 2) trabajo en equipo para ser más eficientes y 3 ) orientación a los resultados. En relación a los temas menos estudiados, se describieron los siguientes: 1) las cualidades perfeccionadas impactan en el DL; 2) la perfección continua de las destrezas y 3 ) los componentes de personalidad representan las aptitudes.

\section{Importantes aportes en relación al desempeño laboral}

En los artículos revisados se encontraron una serie de resultados, aporte o conclusiones referidos a gestión de los recursos humanos. Asimismo, se reconocieron una determinada cantidad de propuestas sobre el DL; los mismos que se caracterizaron y se clasificaron por la cantidad de veces que tuvieron su aparición. Esto es, por cada categoría aparecida, se detalla la cantidad de estudios en que se muestra (ver Tabla 1).

En la Tabla 1 se alcanza a distinguir la existencia de diferentes aportes teóricos/empíricos referidos al DL. Es necesario mencionar que unos fueron abordados en un artículo, en cambio otros fueron mencionado en nueve artículos.

En referencia a la categoría que cuenta con mayor repetición se ubicaron gran cantidad de artículos que determinan que la motivación y la satisfacción laboral impactan positivamente en el DL. Estos mencionan que el recurso humano cumple un rol fundamental para el logro de las metas de una institución, y un buen clima y satisfacción laboral ayudaría en la implementación de dichas estrategias (Montoya et al., 2017).

Por último, nos referimos a la categoría "la motivación, satisfacción laboral, clima 
Tabla 1. Frecuencia de los aportes o propuestas producidos en los estudios

\begin{tabular}{lc}
\hline Categorías & $\begin{array}{c}\mathbf{N}^{\circ} \mathbf{d e} \\
\text { artículos }\end{array}$ \\
\hline La motivación y la satisfacción laboral impactan positivamente en el DL. & 9 \\
Las evaluaciones de DL deben reflejar conocimientos y habilidades. & 4 \\
La gestión del talento humano mejora las competencias laborales. & 4 \\
La capacitación influye favorablemente en el logro competencias laborales. & 3 \\
El reconocimiento de las emociones garantiza un buen DL. & 3 \\
Ambientes laborales propicios que generen satisfacción laboral. & 3 \\
La relación del DL con el tipo de contrato y los cargos son significativas. & 2 \\
El DL se conduce independientemente del clima organizacional. & 2 \\
La participación en la toma de decisiones genera confianza. & 2 \\
El trabajo en equipo mejora el DL. & 2 \\
La relación del DL con el género y la edad de los trabajadores es muy baja. & 1 \\
Los estilos y formas de selección de los decisores no contribuyen al liderazgo. & 1 \\
Modificación de la normatividad de acuerdo a la realidad nacional. & 1 \\
La inteligencia emocional cumple un rol fundamental en el desarrollo de & 1 \\
competencias laborales. & \\
Las aptitudes emocionales transforman el control, comprensión y autogestión. & 1 \\
El clima laboral no se asocia con la incidencia de la violencia laboral. & 1 \\
El uso de la TIC está con el DL. & 1 \\
\hline
\end{tabular}

Del mismo modo, dos artículos con significativa frecuencia está referida a evaluaciones del DL, que deben reflejar conocimientos y habilidades de trabajadores y gestión del talento humano mejorando competencias laborales. Estos estudios también formulan el desarrollo profesional de trabajadores como factor primordial previo a evaluaciones y coloca al recurso humano como eje principal de progreso de una IES.

Igualmente, la categorización realizada en la Tabla 1, ha permitido reflejar dos temas atractivos que debemos considerar. Por un lado, la contribución de temas investigados que aportan puntos de vista teóricos sobre DL. Por otro lado, identificación de temas menos investigados, dentro de ellos mencionamos la violencia laboral, uso de TIC y formas de selección de tomadores de decisión.

También es importante señalar que DL, en resumen, es un factor que se mejora de la siguiente manera: 1) brindando espacios agradables de trabajo; 2) generando confianzay dando aperturaa trabajadores a brindar opiniones; 3) implementación de normas adecuadas a la realidad y que genere beneficios a trabajadores y 4) Trabajar la inteligencia emocional para desarrollar competencias laborales como liderazgo, productividad, comunicación asertiva, compromiso y responsabilidad.

Para terminar esta sección, en tres estudios plantea que la capacitación influye favorablemente en lograr competencias laborales y por consiguiente el DL, el mismo que coincide con Chávez et al.
(2018); Orbe-Guaraca y Ordoñez-Espinoza, (2018) y Rojas y Távara (2017) quienes hacen mención que para mejorar el DL deben trabajar con capacitación en gestión universitaria, tener planes de formación que cuenten con respaldo por parte de superiores para asistir a eventos, lo cual genera una relación estrecha entre jefes y compañeros de trabajo, generando niveles altos de satisfacción laboral.

\section{Discusión}

Se observa diferentes resultados respecto al DL como propósito de estudio, temática que origina muchos espacios de discusión en instituciones privadas y públicas, como mejoramiento de eficiencia y efectividad de trabajadores en labores diarias. Existe la necesidad de gestionar el talento humano y brindar apoyo organizacional, siendo estos factores determinantes en el logro de resultados en IES, ya que su impacto en formación de profesionales es estratégico, quienes serán los motores del desarrollo de una comunidad, región o nación. Coskuner et al. (2018), argumentan que las IES se benefician al tener trabajadores que perciban niveles altos de apoyo organizacional y Chong et al. (2020) afirma que, gestionar el talento humano, entrenarlos y ayudarlos que adquieran técnicas de manejo de emociones, comportamiento, amabilidad, empatía y circunstancias difíciles, pueden ayudar a mejorar resultados.

Al respecto, los artículos encontrados están mayormente enfocados en el rendimiento laboral y clima organizacional. Resaltando su relación con 
el género, cargo laboral, edad y actitud hacia el trabajo para la primera (Calizaya y Bellido, 2019) y segunda variable (Chávez y Chauca, 2020). Con respecto a la edad de trabajadores, Arteche et al. (2020) aseveran que empleados de mayor edad difieren de jóvenes en referencia a facultades físicas y mentales que trae consigo el avance de la edad, lo que impacta en el DL.

Sin embargo, existe otros factores que limitan el espacio de investigación sobre DL, dejando de lado aspectos importantes que inciden positivamente como recompensas, beneficios y trato equitativo en trabajadores que terminan despertando motivación en ellos. Esto coincide con Trujillo et al. (2020), quienes proponen la implementación de sistema de recompensas justas, como sueldos, nivel de ingreso acorde a responsabilidades, reconocimiento por su trabajo, subvenciones, bonos y oportunidades de formación.

Otro aspecto a considerar en el estudio del DL, es la concepción de ser manifestada como descriptor genérico y no como problema; describir interrelaciones no es suficiente, es necesario plantear propuestas que permita dar solución en este campo.

Lo descrito plantea un escenario desafiante, manteniendo muchas dudas sobre el DL que necesitan ser investigadas, planteándose lo siguiente: ¿por qué el modelo disciplinar sigue prevaleciendo? ¿será por desinformación y falta de organización o por otros aspectos que no se puede identificar? Es posible que sean las razones para un limitado desempeño y satisfacción laboral. También existe la posibilidad que en las IES no apliquen estrategias que mejoren la calidad de vida de los trabajadores y por ende de su desempeño. Asimismo, existe la posibilidad que instituciones encargadas de normar servicios laborales en instituciones no estén de acuerdo a expectativas de los trabajadores.

Ante este escenario, existe la posibilidad que los estudios son considerados desde los elementos reactivos, más que proactivos.

\section{Conclusiones}

Los artículos en su mayoría hicieron uso de instrumentos de medición del DL, con análisis genérico, limitado a la descripción de relación de variables y dimensiones e insuficiente peso teórico.
Se ha encontrado que el DL es estudiado como factor desencadenante de la eficacia y eficiencia de los trabajadores de las IES y que los tomadores de decisiones adoptan postura reactiva más que proactiva. Las investigaciones son trabajadas ante situaciones problemáticas institucionales y no, de forma continua y formativa. De este modo, no coadyuva: a) en la motivación a trabajadores para mejorar el DL; b) desarrollar las destrezas, habilidades y aptitudes personales y sociales; y c) elevar la satisfacción laboral que promueva mejorar el DL.

Es importante indicar, el encuentro de algunas limitaciones en el desarrollo del presente estudio, que deben tomar en cuenta los investigadores en adelante. Primero, la limitada cantidad de artículos referidos al DL que investigan de manera profunda aspectos innovadores para mejorar el rendimiento laboral, clima organizacional, inclusive la cultura organizacional. Segundo, la mayoría son investigaciones correlacionales que solo presentan resultados y conclusiones, no brindan aportes que contribuya al desarrollo epistemológico. Y tercero, al desarrollarse este estudio enmarcado en la zona geográfica latinoamericana, ha limitado a tener una concepción, filosofía, resultados, aportes y conclusiones de investigadores de otros continentes.

Es necesario continuar las investigaciones sobre DL considerando algunas interrogantes: 1) en qué forma la academia (universidad o institutos técnicos) contribuye la formación de profesionales con perfiles que potencien el talento humano; 2) qué viene interesando a gestores del talento humano para mejorar el DL; 3) será que las IES no la tienen muy clara o no están en contexto actual de las necesidades; 4) cómo se consigue que sea trabajada de forma sostenible y predictiva; y 5) cómo colaborar con la epistemología desde componentes que predominen y qué aspectos propositivos considerar para disminuir las consecuencias de estos hacia el DL.

\section{Referencias bibliográficas}

Al-Kurdi, O. F., El-Haddadeh, R. \& Eldabi, T. (2020). The role of organisational climate in managing knowledge sharing among academics in higher education. International Journal of Information Management, 50(2020), 217-227. 10.1016/j.ijinfomgt.2019.05.018 
Álvarez-Indacochea, B., Indacochea-Ganchozo, B., Álvarez-Indacochea, A., Yoza-Rodriguez, N. y Figueroa-Soledispa, M. (2018). La evaluación del desempeño laboral en la Educación Superior. Dominio de Las Ciencias, 4(1), 362-372. 10.23857/dc.v4i1.749

Álvarez-Indacochea, B., Indacochea, B., Yoza, N. y Alavarez-Indacochea, A. (2018). Modelo de evaluación del desempeño laboral para el personal administrativo universitario. Rev. SINAPSIS, 1(12). 10.37117/s.vli12.140

Álvarez, B., Alfonso, D. y Indacochea, B. (2018). El desempeño laboral: Un problema social de la ciencia. Didasc@lia: Didáctica y Educación, IX(2), 147-158.

Arteche, D., Almazan, J. \& Adolfo, C. (2020). Functional Capability and Job Competence of Part-Time Postretiree Workers in Higher Academic Institutions. Ageing International, 45(3), 305-314. 10.1007/s12126-019-09357-7

Bohórquez, E., Pérez, M., Caiche, W. y Benavides, A. (2020). La Motivación y el Desempeño Laboral: El Capital Humano como factor clave en una organización. Universidad y Sociedad, 12(3), 285-390.

Bravo, G., Álava, W. y Álava, E. (2021). Docentes Universitarios y su desempeño laboral. Serie Científica de La Universidad de Las Ciencias Informáticas, 14(3), 167-176.

Calizaya, J. y Bellido, R. (2019). Clima organizacional y rendimiento laboral en servidores administrativos de una universidad pública de Arequipa. Revista de Psicología, 9(3), 37-55. 10.36901/psicologia.v9i3.701

Campbell,J.P.\&Wiernik,B.M.(2015). TheModeling and Assessment of Work Performance. In Annual Review of Organizational Psychology and Organizational Behavior (Vol. 2). 10.1146/ annurev-orgpsych-032414-111427

Chávez, A., Moscoso, M. y Manrique, Z. (2018). Capacitación en gestión universitaria para lograr competencias laborales en el personal administrativo de la Universidad Nacional Autónoma Altoandina de Tarma, 2017. Horizonte de La Ciencia, 8(14), 131-139.

Chávez, R. y Chauca, P. (2020). Liderazgo y clima organizacional en docentes del área de la salud. REICE: Revista Electrónica de Investigación En Ciencias Económicas, 8(16), 40-53. 10.5377/reice.v8i16.10659

Chiang, M. y Ojeda, J. (2013). Estudio de la relación entre satisfacción laboral y el desempeño de los trabajadores de las ferias libres. Contaduría y
Administración, 58(2), 39-60. 10.1016/s01861042(13)71209-9

Chiavenato, I. (2009). Comportamiento organizacional: la dinámica del éxito en las organizaciones (México: Mc Graw Hill Interamericana Editores. 2da. Ed. México: McGraw-Hill.

Chong, S. C., Falahat, M. \& Lee, Y. S. (2020). Emotional intelligence and job performance of academicians in Malaysia. International Journal of Higher Education, 19(1), 69-80. 10.5430/ijhe.v9n1p69

Coskuner, S., Costur, R., Bayhan-Karapinar, P., Metin-Camgoz, S., Ceylan, S., DemirtasZorbaz, S., Aktas, E. F. \& Ciffiliz, G. (2018). Mobbing, organizational identification, and perceived support: Evidence from a higher education institution. Egitim Arastirmalari - Eurasian Journal of Educational Research, 2018(73), 19-40. 10.14689/ejer.2018.73.2

Cubero, H., Thamara Visbal, E. y Olivar, J. (2018). Propuesta para gestionar cambios de evaluación del desempeño. Caso: Universidad Simón Bolívar. Orbis: Revista Científica Ciencias Humanas, 13(39), 19-34.

Dermawan, R. \& Handayani, W. (2019). Factors triggering organizational citizenship behavior, failure and success: A case study of a higher education institution. Humanities and Social Sciences Reviews, 7(1), 156-163. 10.18510/ hssr.2019.7119

Duque, J., García, M. y Hurtado, A. (2017). Influencia de la inteligencia emocional sobre las competencias laborales: un estudio empírico con empleados del nivel administrativo. Estudios Gerenciales, 33(144), 250-260. 10.1016/i.estger.2017.06.005

Eirín, R., Sanmiguel-Rodríguez, A. y Rodríguez Rodríguez, J. (2020). Las razones del cambio de desempeño laboral en los docentes de Educación Física en el contexto gallego. Retos, 2041(41), 153-161. 10.47197/retos.v0i41.81815

Foy, T., Dwyer, R. J., Nafarrete, R., Hammoud, M. S. S. \& Rockett, P. (2019). Managing job performance, social support and worklife conflict to reduce workplace stress. International Journal of Productivity and Performance Management, 68(6), 1018-1041. 10.1108/IJPPM-03-2017-0061

Fulthorp, K. \& D'Eloia, M. H. (2015). Managers' Perceptions of Entry-Level Job Competencies When Making Hiring Decisions for Municipal Recreation Agencies. Journal of Park \& 
Recreation Administration, 33(1), 57-71.

Grobler, A. \& Van Rensburg, M. J. (2019). Organisational climate, person-organisation fit and turn over intention: a generational perspective within a South African Higher Education Institution. Studies in Higher Education, 44(11), 2053-2065. 10.1080/03075079.2018.1492533

Hernández, J. y Morales, J. (2017). Evaluación de la motivación y satisfacción laboral en un organismo autónomo de la administración pública del Estado de Sinaloa. Daena: International Journal of Good Conscience, 12(2), 107-147.

Ivancevich, J., Konopaske, R. y Matteson, M. (2006). Comportamiento Organizacional. 7ma Ed. México: McGraw-Hill.

Jyoti, J. \& Sharma, P. (2017). Empirical investigation of a moderating and mediating variable in between mentoring and job performance: A structural model. Journal of Work and Organizational Psychology, 33(1), 55-67. 10.1016/j.rpto.2017.01.002

Khan, M. N., Malik, S. A. \& Janjua, S. Y. (2019). Total Quality Management practices and work-related outcomes: A case study of higher education institutions in Pakistan. International Journal of Quality and Reliability Management, 36(6), 864-874. 10.1108/IJQRM-04-2018-0097

Medina-Garrido, J. A., Biedma-Ferrer, J. M. \& Ramos-Rodríguez, A. (2017). Relationship between work-family balance, employee well-being and job performance. Academia Revista Latinoamericana de Administración, 30(1), 40-58. 10.1108/ARLA-08-2015-0202

Mohd Ali, H., Ayodele, L. A. \& Ibrahim, M. B. (2019). The future sustainability of malaysian higher education institutions: What matters most? Asian Academy of Management Journal, 24(Supp. 1), 171-186. 10.21315/ aamj2019.24.s1.12

Moher, D., Liberati, A., Tetzlaff, J., Altman, D. G. y PRISMA Group, T. (2014). Items de referencia para publicar Revisiones Sistemáticas y Metaanálisis: La Declaración PRISMA. Revista Española de Nutrición Humana y Dietética, 18(3), 172. 10.14306/renhyd.18.3.114

Montoya, P., Bello-Escamilla, N., Bermúdez, N., Burgos, F., Fuentealba, M. y Padilla, A. (2017). Satisfacción Laboral y su Relación con el Clima Organizacional en Funcionarios de una Universidad Estatal Chilena. Ciencia
\& Trabajo, 19(58), 7-13. 10.4067/S071824492017000100007

Musah, M., Ali, H., Vazhathodi, S., Tahir, L., Daud, K., Said, H. \& Kamil, N. (2016). Organisational climate as a predictor of workforce performance in the Malaysian higher education institutions. Quality Assurance in Education, 24(3), 416-438. 10.1108/QAE-10-2014-0048

Orbe-Guaraca, M.-P. y Ordoñez-Espinoza, C.G. (2018). Elaboración de un sistema de acciones para la motivación en el desempeño laboral del personal docente, administrativo y de servicio de la Universidad Católica de Cuenca, Extensión Cañar. 3C Empresa: Investigación y Pensamiento Crítico, 7(3), 1027. $10.17993 / 3$ cemp. 2018.070335.10-27

Pa'wan, F. \& Omar, R. (2018). Effects of innovative organizational climate on organizational commitment in Malaysian higher education institutions. Malaysian Online Journal of Educational Management, 6(2), 1-21. 10.22452/mojem.vol6no2.1

Panduro, L., Bollet, F., Ramírez, L. y Chenet, M. (2019). Desempeño laboral y gestión administrativa de la Universidad Privada de Pucallpa, 2018-2019. Cultura Viva Amazónica, 4(2), 47-51. 10.37292/riccva.v4i2.149

Pantoja-Pantoja, A., Navarrete-Acosta, A., Zambrano-Guerrero, C. y MatabanchoyTulcán, S. (2020). Relación entre clima organizacional y violencia laboral en funcionarios de una universidad pública. Universidad y Salud, 22(2), 137-147. 10.22267/ rus.202202.185

Pedraza, N. (2020). Satisfacción laboral y compromiso organizacional del capital humano en el desempeño en instituciones de educación superior. Revista Iberoamericana Para La Investigación y El Desarrollo Educativo, 10(20). 10.23913/ride.v10i20.595

Pongton, P. \& Suntrayuth, S.(2019). Communication satisfaction, employee engagement, job satisfaction, and job performance in higher education instituti. ABAC Journal, 39(3), 90110.

Pujol-Cols, L. y Dabos, G. (2018). Satisfacción laboral: una revisión de la literatura acerca de sus principales determinantes. Estudios Gerenciales, 34(146), 3-18. 10.18046/j. estger.2018.146.2809

Ramos-Villagrasa, P. J., Barrada, J. R., FernándezDel-Río, E. \& Koopmans, L. (2019). 
Assessing job performance using brief selfreport scales: The case of the individual work performance questionnaire. Journal of Work and Organizational Psychology, 35(3), 195205. 10.5093/jwop2019a21

Reyes, H. (2020). Artículos de Revisión. Revista Médica de Chile, 148, 103-108.

Rodríguez-Marulanda, K. y Lechuga-Cardozo, J. (2019). Desempeño laboral de los docentes de la Institución Universitaria ITSA. Rev. esc.adm.neg., 87, 79-101. 10.21158/01208160. n87.2019.2452

Rodriguez, R., Vásquez, M., Flores, I., Flores, R., Ramírez, S., Martínez, F., Aguilar, E. y Garnica, M. (2015). Factores explicativos del nivel de satisfacción laboral de los trabajadores de los institutos de ciencias básicas e ingeniería y ciencias de la salud de la universidad autónoma del estado de hidalgo, una evidencia empírica. XIKUA Boletín Científico de La Escuela Superior de Tlahuelilpan, 3(5), 1-10. 10.29057/xikua. v3i5.1273

Rojas, W. y Távara, Ú. (2017). Cultura organizacional y satisfacción laboral en los trabajadores de la Universidad Marcelino Champagnat. Revista EDUCA UMCH, 10, 177-200. 10.35756/educaumch.201710.22

Sánchez, A. (2011). Manual de redacción académica e investigativa: cómo escribir, evaluar y publicar artículos. 1ra Ed. Colombia: Fundación Universitaria Católica del Norte.

Sora, B., Höge, T., Caballer, A., Peiró, J. y Boada, J. (2021). Job Insecurity and Performance:
The Mediating Role of Organizational Justice in Terms of Type of Contract. Psicothema, 33(1), 86-94. 10.7334/psicothema2020.205

Ticona, J. (2020). Uso de las TIC y su relación con el desempeño laboral del personal administrativo de las universidades nacionales. Revista de Investigaciones de La Escuela de Posgrado, 9(3), 1782-1791.

Trujillo, P., Ortiz, M., Carrasco, M., Herrera, G. y Martínez, C. (2020). Satisfacción laboral y desempeño académico universitario. InterCambios. Dilemas Y Transiciones De La Educación Superior, 7(2), 77-84.

Vargas, S. y Flores, M. (2019). Cultura organizacional y satisfacción laboral como predictores del desempeño laboral en bibliotecarios. Investigación Bibliotecológica: Archivonomía, Bibliotecología Información, 33(79), 149-176. 10.22201/ iibi.24488321xe.2019.79.57913

Zapata, G. y Rueda, M. P. (2018). Teoría de dependencia de recursos: premisas $\mathrm{y}$ aplicaciones. Ciencia y Sociedad, 43(1), 7592. 10.22206/cys.2018.v43i1.pp75-92

Zuta, N., Castro, L. y Zela, L. (2018). Motivación profesional y desempeño laboral de los docentes de práctica clínica de la escuela profesional de enfermería. Investigación Valdizana, 12(3), 157-164. 10.33554/ riv.12.3.151 